\title{
Energy levels of rare-earth ions in crystal lattice sites of cubic symmetry
}

\author{
C.A.J. Ammerlaan
}

Van der Waals-Zeeman Institute, University of Amsterdam, Valckenierstraat 65, 1018 XE Amsterdam, The Netherlands

\begin{abstract}
Numerical calculations are presented for the energy levels of the rare-earth ion $\mathrm{Er}^{3+}$ in a crystalline field of cubic symmetry. A distinction is made between the five different point groups within the system of cubic symmetry. Traditionally only the point groups $O, O_{h}$ and $T_{d}$ are considered. As an extension, in the present analysis the effect of an additional crystalfield term appearing for the cubic point groups $T$ and $T_{h}$ is explored.
\end{abstract}

Keywords: $\mathrm{Er}^{3+}$, rare-earth ions, crystal-field theory.

Paper received 28.12.02; accepted for publication 18.03.03.

\section{Introduction}

Rare-earth elements are dopants donating interesting and often useful properties to host crystals. In many cases, such as in optical absorption or light emission, there exists a direct relation to the energies in ground and excited states of the electron system. As an example, typical of rare-earth impurities in general, energy levels of the ion of erbium in threefold ionized state will be considered. Ion $\mathrm{Er}^{3+}$ has electronic configuration $4 f^{11}$ and, following Hund's rule of level occupation, possesses an orbital momentum $L=6$ and spin $S=3 / 2$. Spin-orbit interaction is strong for these high- $\mathrm{Z}$ ions and forms the largest correction on the energies as resulting from Coulomb electrostatic fields. By the spin-orbit energy $\lambda \mathbf{L} \cdot \mathbf{S}$ the degeneracy of the 52 states will be partially lifted. For erbium, four levels characterized by total angular momentum $J$ ranging from $J=L-S=9 / 2$ to $J=L+S=15 / 2$ will be formed. With the negative spin-orbit coupling constant $\lambda$ the state labeled ${ }^{4} I_{15 / 2}$ forms the ground state. It is separated from the next higher state ${ }^{4} I_{13 / 2}$ by about $800 \mathrm{meV}$. Calculations to be reported in this paper are restricted to the ground state, which is considered as isolated and is treated in the spin $J$ formalism.

\section{Crystal-field potentials and operators}

When the rare-earth ion is incorporated as an impurity in a crystal, the ground state ${ }^{4} I_{15 / 2}$, with still 16 fold degeneracy, is further split by the crystal field. The precise effect depends on symmetry and strength of the crystal field. For the lower symmetries, such as trigonal or orthorhombic, the maximum splitting into eight Kramers doublets will occur. In a cubic field, as will be considered further, the splitting is into three quartets and two doublets. The crystal field is represented by its equivalent spin operator $H$. It is constructed from expressions of potential which are invariant in the crystal field considered. For cubic symmetries the possible forms of potential, up to sixth order, are given in Table 1. Expressions of the odd order 3 and 5 will lead to vanishing matrix elements within the basis of $4 f$ states and can be omitted. One is left with the relevant potentials and operators of fourth and sixth order as specified in Table 1. In the majority of literature only the fourth-order operator $\mathrm{O}_{4}{ }^{0}+$ $5 \mathrm{O}_{4}{ }^{4}$ and the sixth-order operator $\mathrm{O}_{6}{ }^{0}-21 \mathrm{O}_{6}{ }^{4}$ are considered $[1,2]$. These are the required terms for the most frequently occurring point groups $O, O_{h}$ and $T_{d}$. For the point groups of the lower symmetry in the cubic system 


\section{C.A.J. Ammerlaan: Energy levels of rare-earth ions in ...}

Table I. Summary of invariant potentials and corresponding crystal-field operators, up to the sixth order, for five point groups of cubic symmetry.

\begin{tabular}{lll}
\hline \hline Point groups & Potential & Operator \\
\hline$T, T_{d}, T_{h}, O, O_{h}$ & $+x^{2}+y^{2}+z^{2}$ & $+J(J+1)$ \\
\hline$T, T_{d}$ & $+x y z$ & $+O_{4}^{0}+5 O_{4}{ }^{4}$ \\
\hline$T, T_{d}, T_{h}, O, O_{h}$ & $+x^{4}+y^{4}+z^{4}-3\left(x^{2} y^{2}+y^{2} z^{2}+z^{2} x^{2}\right)$ & \\
\hline$T, T_{d}$ & $+x y z\left(x^{2}+y^{2}+z^{2}\right)$ & $+O_{6}{ }^{0}-21 O_{6}{ }^{4}$ \\
\hline$T, T_{d}, T_{h}, O, O_{h}$ & $+2\left(x^{6}+y^{6}+z^{6}\right)-15\left(x^{4} y^{2}+y^{4} z^{2}+z^{4} x^{2}+x^{4} z^{2}+y^{4} x^{2}+z^{4} y^{2}\right)+180 x^{2} y^{2} z^{2}$ & $+O_{6}{ }^{2}-O_{6}{ }^{6}$ \\
\hline$T, T_{h}$ & $+\left(x^{4} y^{2}+y^{4} z^{2}+z^{4} x^{2}\right)-\left(x^{4} z^{2}+y^{4} x^{2}+z^{4} y^{2}\right)$ & \\
\hline
\end{tabular}

an additional form of sixth-order potential will be allowed and the corresponding operator $\mathrm{O}_{6}{ }^{2}-\mathrm{O}_{6}{ }^{6}$ will be present $[3,4]$. Numerical calculations were made with this more general form of crystal-field operator. Matrix elements were calculated in the basis set of 16 spin states in ${ }^{4} I_{15 / 2}$. Energies were obtained as the eigenvalues of the $16 \times 16$ matrices. The level splittings induced by the crystal field, with an order of magnitude of $50 \mathrm{meV}$, are small compared to the separations between spin-orbit levels.

\section{Crystal-field energies}

For the general cubic field the corresponding Hamiltonian operator is formulated as

$$
H=+(\cos \alpha) O_{4}+(\sin \alpha)\left[(\cos \beta) O_{6,1}+(\sin \beta) O_{6,2}\right] .
$$

Parameter $\alpha$ specifies the relative weights of fourthand sixth-order energies, whereas parameter $\beta$ details the type of the latter potential. Expressions of the basic operators are given as

$$
\begin{aligned}
& O_{4}{ }^{0}=+35 J_{z}^{4}-30 J(J+1) J_{z}{ }^{2}+25 J_{z}^{2}-6 J(J+1)+ \\
& +3 J^{2}(J+1)^{2} \\
& O_{4}{ }^{4}=+(1 / 2)\left(J_{+}{ }^{4}+J_{-}^{4}\right) \\
& O_{6}{ }^{0}=+231 J_{z}{ }^{6}-315 J(J+1) J_{z}{ }^{4}+735 J_{z}{ }^{4}+ \\
& +105 J^{2}(J+1)^{2} J_{z}{ }^{2}-525 J(J+1) J_{z}{ }^{2}+294 J_{z}{ }^{2} \\
& -5 J^{3}(J+1)^{3}+40 J^{2}(J+1)^{2}-60 J(J+1) \\
& O_{6}{ }^{2}=+(1 / 4)\left\{\left[+33 J_{z}{ }^{4}-18 J(J+1) J_{z}{ }^{2}-123 J_{z}{ }^{2}+\right.\right. \\
& \left.+J^{2}(J+1)^{2}+10 J(J+1)+102\right]\left(J_{+}{ }^{2}+J_{-}{ }^{2}\right)+ \\
& +\left(J_{+}{ }^{2}+J_{-}^{2}\right)\left[+33 J_{z}{ }^{4}-18 J(J+1) J_{z}{ }^{2}-123 J_{z}{ }^{2}+\right. \\
& \left.\left.+J^{2}(J+1)^{2}+10 J(J+1)+102\right]\right\} \\
& O_{6}{ }^{4}=+(1 / 4)\left\{\left[+11 J_{z}{ }^{2}-J(J+1)-38\right]\left(J_{+}{ }^{4}+J_{-}^{4}\right)+\right. \\
& \left.+\left(J_{+}{ }^{4}+J_{-}^{4}\right)\left[+11 J_{z}{ }^{2}-J(J+1)-38\right]\right\}
\end{aligned}
$$

and

$O_{6}{ }^{6}=+(1 / 2)\left(J_{+}{ }^{6}+J_{-}^{6}\right)$.
Operators appearing in the crystal-field Hamiltonian are the combinations

$$
\begin{aligned}
& O_{4}=\left(O_{4}{ }^{0}+5 O_{4}{ }^{4}\right) / F_{4}, \\
& O_{6,1}=\left(O_{6}{ }^{0}-21 O_{6}{ }^{4}\right) / F_{6,1}
\end{aligned}
$$

and

$$
O_{6,2}=\left(O_{6}^{2}-O_{6}^{6}\right) / F_{6,2} \text {. }
$$

Actual calculations were performed with scaling factors $F_{4}=60$ and $F_{6,1}=13860$, as usual, and $F_{6,2}=1260$. Always five levels are obtained: two doublets belonging to the $\Gamma_{6}$ and $\Gamma_{7}$ irreducible representations, respectively, and three quartets of $\Gamma_{8}$ symmetry type. The traditional result, obtained by putting parameter $\beta=0^{\circ}$, is given in Fig. 1. Although presented in a different format this re-

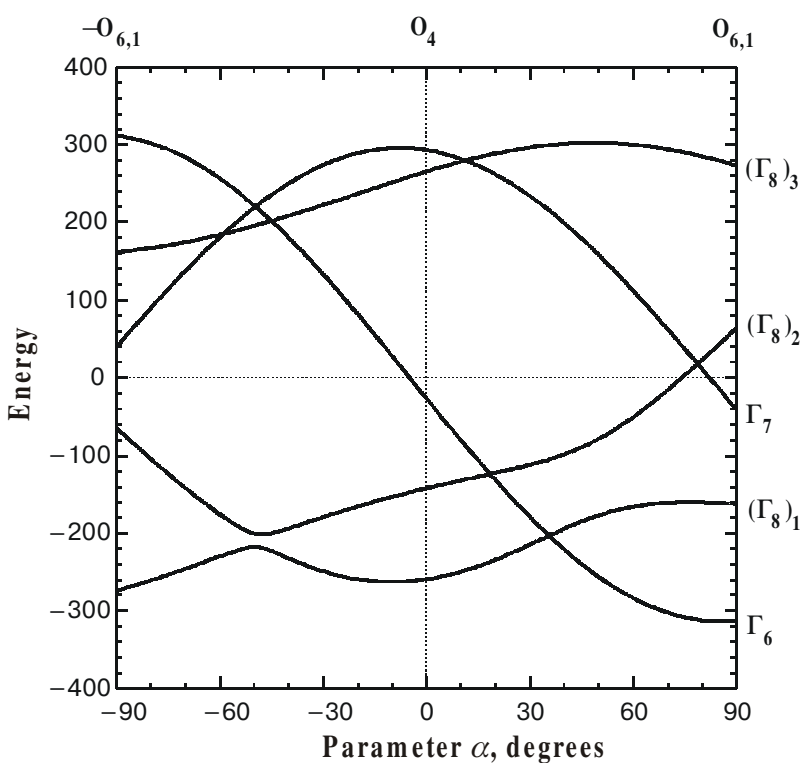

Fig. 1. Crystal-field energies, in $\mathrm{cm}^{-1}$, of doublets $\Gamma_{6}$ and $\Gamma_{7}$ and of the quartets $\left(\Gamma_{8}\right)_{i}, i=1,2$ and 3 , representing the eigenvalues of equation (1). Parameter $\alpha$ in the range $-90^{\circ}<\alpha<+90^{\circ}$ controls the mixing of the fourth-order crystal field $O_{4}$ with type-1 sixth-order crystal field $O_{6,1}$. Parameter $\beta=0^{\circ}$. 


\section{C.A.J. Ammerlaan: Energy levels of rare-earth ions in ...}

sult is entirely equivalent to the data as given in the classical paper of Lea, Leask and Wolf [1]. Several spectra as observed in optical experiments were interpreted in this diagram. One expects this diagram to be appropriate for small defects of simple structure not degrading the crystal point group symmetry. This will be the case, for instance, for single atom impurities in a substitutional site or an interstitial $T_{d}$ site in a diamond crystal structure. Fig. 2 shows the results as calculated for the sixthorder field $O_{6,2}$ of the point groups $T$ and $T_{h}$ by specifying $\beta=+90^{\circ}$. It deviates in a substantial manner from the previous result for $O_{6,1}$. Such a diagram will have to be considered for defect complexes of lower symmetry only leaving the $T$ and $T_{h}$ symmetry operations intact. To author's knowledge, no such analyses have been presented in the literature yet, there are no sets of experimental data obtained that show the clear need for the lower-symmetry analysis. The transition from pure $O_{6,1}$ to pure $O_{6,2}$ potential is illustrated in Fig. 3. In the real situation one expects a mixture of sixth-order potentials of types 1 and 2 to be present for the $T$ - and $T_{h}$-type of centers. It may be assumed that the $O_{6,1}$ potential will be the leading term, and that only a small admixture of the $O_{6,2}$ type of potential will occur. This case represented by a small value of parameter $\beta$ is illustrated by Fig. 4 for $\beta=+10^{\circ}$. Changes with respect to Fig. 1 are observed to be small, except for the regions of level crossings and anti-crossings.

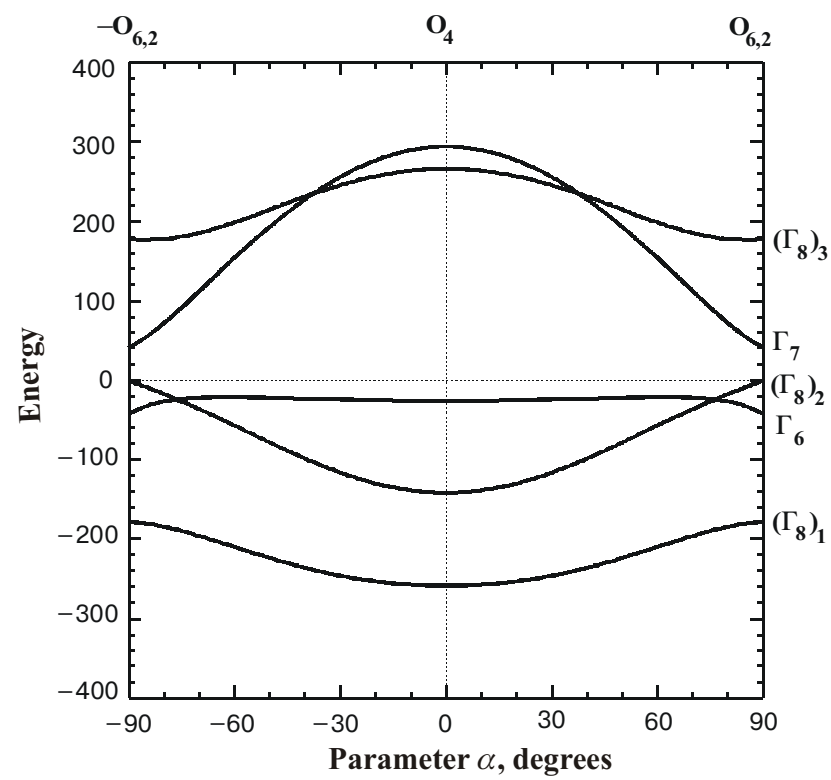

Fig. 2. Crystal-field energies, in $\mathrm{cm}^{-1}$, of doublets $\Gamma_{6}$ and $\Gamma_{7}$ and of the quartets $\left(\Gamma_{8}\right)_{i}, i=1,2$ and 3 , representing the eigenvalues of equation (1). Parameter $\alpha$ in the range $-90^{\circ}<\alpha<+90^{\circ}$ controls the mixing of the fourth-order crystal field $O_{4}$ with type-2 sixth-order crystal field $O_{6,2}$. Parameter $\beta=+90^{\circ}$.

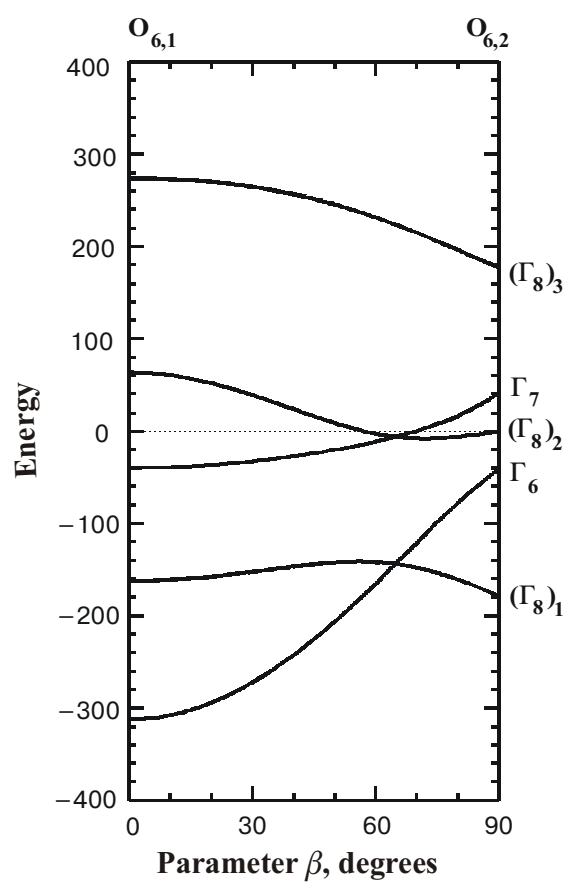

Fig. 3. Crystal-field energies, in $\mathrm{cm}^{-1}$, of the five levels in cubic symmetry for the transition from type-1 to type-2 sixth-order potential. Parameter $\alpha=+90^{\circ}$.

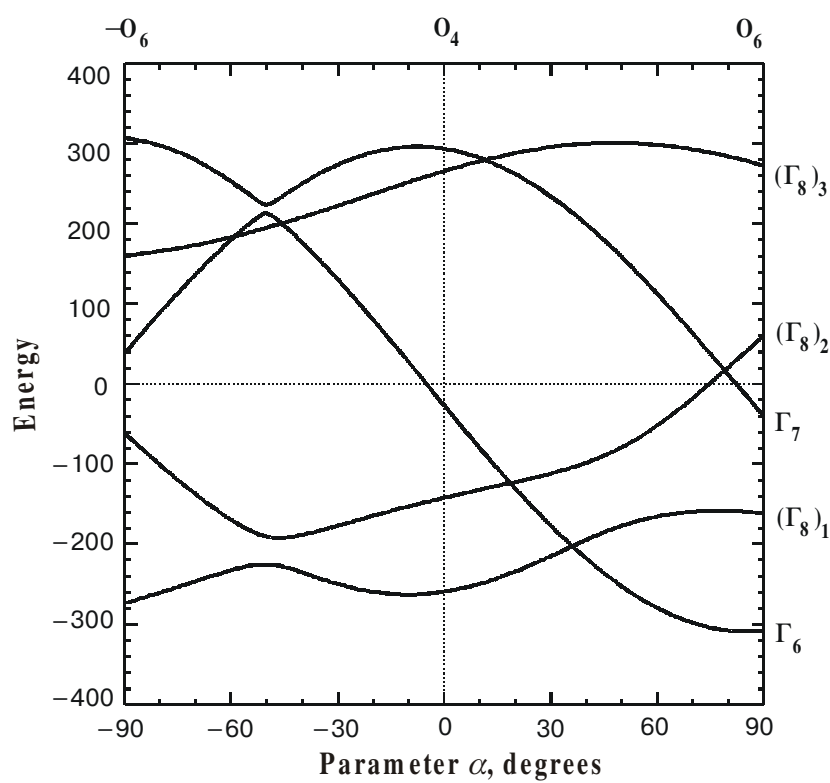

Fig. 4. Crystal-field energies, in $\mathrm{cm}^{-1}$, of doublets $\Gamma_{6}$ and $\Gamma_{7}$ and of the quartets $\left(\Gamma_{8}\right)_{i}, i=1,2$ and 3 , representing the eigenvalues of equation (1). Parameter $\alpha$ in the range $-90^{\circ}<\alpha<+90^{\circ}$ controls the mixing of fourth-order and sixth-order crystal fields. A modest presence of the lower-symmetry sixth-order field for centers of $T$ or $T_{h}$ symmetry is represented by parameter $\beta=+10^{\circ}$. 


\section{C.A.J. Ammerlaan: Energy levels of rare-earth ions in ...}

\section{Conclusion}

Using a numerical scheme of computations, the energy levels of rare-earth centers of cubic symmetry in crystalline hosts are calculated. It is demonstrated that the energy level diagram for centers of the point group symmetries $T$ and $T_{h}$ can differ substantially from that for the point groups $O, O_{h}$ and $T_{d}$.

\section{Acknowledgement}

The author is grateful to Prof. M.M. Zaripov of Kazan State University for bringing the subject to his attention.

\section{References}

1. K.R. Lea, M.J.M. Leask and W.P. Wolf, J. Phys. Chem. Solids 23, 1381 (1962)

2. A. Abragam and B. Bleaney, Electron Paramagnetic Resonance of Transition Ions, Clarendon Press, Oxford, UK, 1970.

3. S.A. Al'tshuler and B.M. Kozyrev, Electron Paramagnetic Resonance in Compounds of Transition Elements, John Wiley \& Sons, New York, USA, 1974.

4. A.M. Leushin, Paramagnitnyi Rezonans, Kazan University, Kazan, Russia, 1964 (in Russian). 